\title{
Fair Curve Networks in Nonlinear Geometries
}

\author{
Johannes Wallner* \\ Vienna University of Technology
}

\author{
Helmut Pottmann ${ }^{\dagger}$ \\ Vienna University of Technology
}

\author{
Michael Hofer ${ }^{\ddagger}$ \\ ECE Dept., University of Minnesota
}

\begin{abstract}
Fair curve networks in nonlinear geometries are an extension of our work [Hofer and Pottmann 2004] on energy-minimizing spline curves in manifolds. Instead of designing with one single curve, we here present a variational approach for the design with an entire network of curves. After a geometric characterization of fair, i.e., energy-minimizing curve networks, we discuss their discretization and computation. Fair curve networks in nonlinear geometries have a variety of applications including surface parameterization, aesthetic remeshing and design of fair surfaces in the presence of obstacles.
\end{abstract}

\section{Characterization and Computation}

A curve network $\mathscr{N}$ consists of a finite set $C$ of curves $c$, each of them defined in its parameter interval $\left[a_{c}, b_{c}\right]$. If we replace the set of curves $C$ by a set of polygons $P$ we get a polygon network, again denoted by $\mathscr{N}$. 'Knots' are points $k$ of $S$ that are contained in at least two different curves/polygons of $\mathscr{N}$, see Fig. 1. The knots together with the curves/polygons define the connectivity of the network. A polygon network $\mathscr{N}$ can represent a triangle mesh if every vertex is a knot and we have three sets of polygons as shown in Fig. 1. For a single curve $c$ we define energies $E_{1}, E_{2}, E_{\tau}$ by

$$
E_{1}(c)=\int_{a_{c}}^{b_{c}}\|\dot{c}(t)\|^{2} d t, \quad E_{2}(c)=\int_{a_{c}}^{b_{c}}\|\ddot{c}(t)\|^{2} d t, \quad E_{\tau}=E_{2}+\tau E_{1},
$$

where $\tau$ is a tension parameter. The energy of the entire network $\mathscr{N}$ is the sum of energies of the single network curves $c$ : $E(C)=\Sigma_{c \in C} E(c)$, where $E$ is one of the energies of (1). We consider networks constrained to a surface $S$, and networks which must not enter an obstacle.
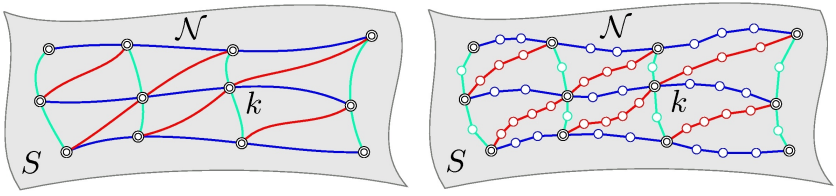

Figure 1: Red, green and blue curves/polygons of a network $\mathscr{N}$ on a surface $S$. Knots are marked with $\odot$.

New theoretic results extend the characterization of energy-minimizing curves from [Hofer and Pottmann 2004] to fair curve networks. There we have shown that e.g. $E_{2}$-minimizing curves in a surface $S$ have 4-th derivative vectors orthogonal to $S$. The geometric characterization of networks is in the spirit of the curve case: Away from knots, we have the curve conditions again, together with a balance condition at each knot. The most important fairness property is that each curve of $\mathscr{N}$, which originally is required to be smooth only, is actually $C^{2}$.

An unconstrained $E_{2}$-minimizing curve network turns out to consist of $C^{2}$ cubic splines with natural end conditions and additional balance conditions at the knots. The usually unique $E$-minimal curve

\footnotetext{
*e-mail:wallner@geometrie.tuwien.ac.at

†email:pottmann@geometrie.tuwien.ac.at

†email:hofer@geometrie.tuwien.ac.at
}
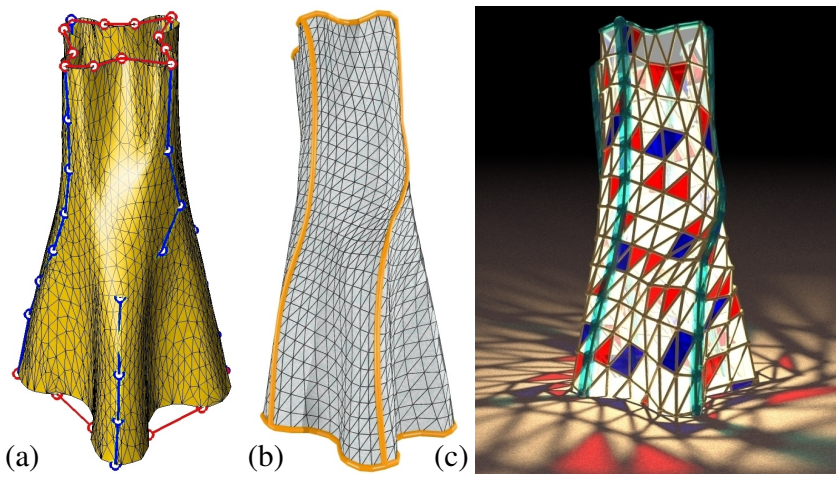

Figure 2: (a) User input on original triangle mesh. (b) Fair mesh $\mathscr{N}$ interpolating the given input polygons. (c) Design based on coarser fair mesh interpolating the given input polygons.

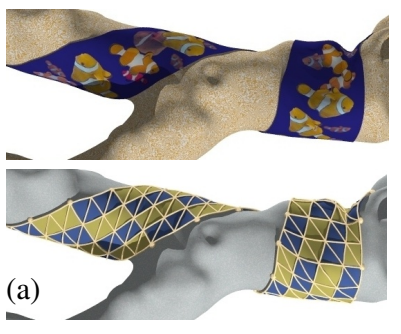

(b)

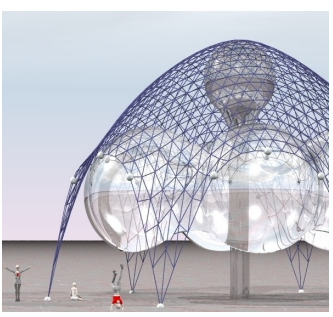

Figure 3: (a) Fair polygon network used for texture mapping. (b) Architectural design from curve network avoiding obstacles.

network in the unconstrained case is found as solution of a system of linear equations.

For computation in the constrained case, we minimize a discrete energy of a polygon network with a nonlinear conjugate gradient algorithm. As in the single curve case, fair curve networks can be computed on various surface representations (parametric, implicit, mesh or point set). We have implemented fair polygon networks (FPNs) with different topologies including 'patch' (Fig. 3) and 'cylinder' (Fig. 2). Total computation times per vertex for the examples shown are about $0.01 \mathrm{~s}$ on a $2 \mathrm{GHz} \mathrm{PC}$.

FPNs are well suited to compute visually pleasing meshes in the sense that they are formed by sequences of fair discretized curves. By fixing certain knots or even some curves, the designer can influence the creation of a fair mesh (see Fig. 2).

FPNs may also be applied to surface parameterization by mapping chosen lines of the parameter domain to fair curves on a surface. For instance, it is easy to wind a visually pleasing textured band around an object (see Fig. 3a).

Further, FPNs are useful for the design of fair surfaces which avoid given obstacles (Fig. 3b).

\section{References}

Hofer, M., And Pottmann, H. 2004. Energy-minimizing splines in manifolds. ACM Trans. Graphics 23, 3, 284-293. 